\title{
Flexible Lösung, die Kosten reduziert
}

Bei Lohnfertigern und Unternehmen mit einem schnell wechselnden Teilespektrum sind regelmäßig Investitionen in Reinigungsbehältnisse erforderlich. Für diese Anwendung wurden spezielle Einsätze entwickelt, die es ermöglichen, die Boxen schnell und kostengünstig anzupassen. Und das selbst dann, wenn die Reinigungsbehältnisse bisher für Schüttgut genutzt wurden.

$B_{b}^{e}$ ei der Reinigung von Präzisionsbauteilen sind üblicherweise hohe Sauberkeitsanforderungen zu erfüllen. Dies gelingt meist nur durch den Einsatz teilespezifisch gestalteter Reinigungsbehältnisse. Allerdings machen kürzere Produktlebenszyklen, die weiter zunehmende Variantenvielfalt oder ein schnell wechselndes Teilespektrum den Austausch der an die Werkstückgeometrie angepassten Lösungen in immer kürzeren Zeitabständen erforderlich. Daraus resultieren hohe Investitionskosten für Reinigungsbehältnisse, die sich auf die Stückkosten und damit auf die Wettbewerbsfähigkeit auswirken.

Abhilfe schafft in diesen Fällen eine flexible und kostengünstige Lösung von Metallform Wächter. Sie besteht aus zwei Typen von Einsätzen, die an

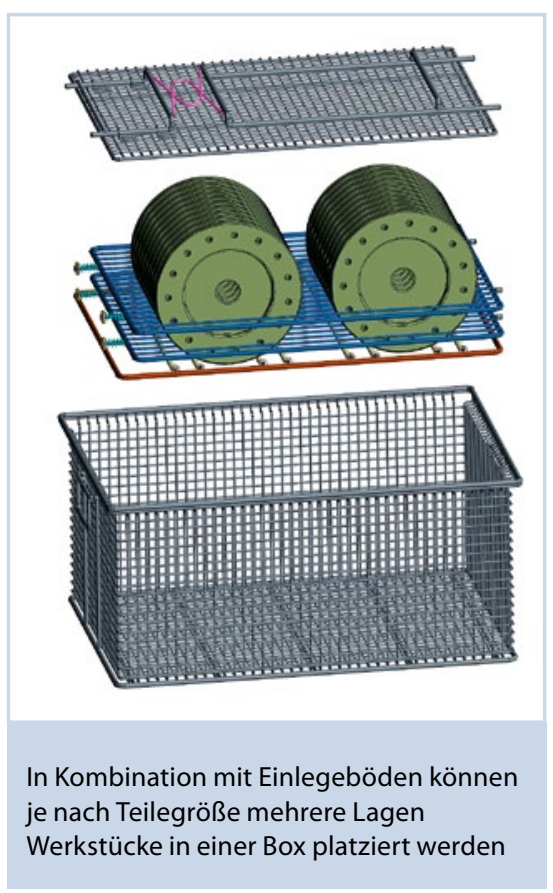

die Teileabmessungen angepasst werden. Eine Einsatzart gibt die Anzahl der Fachreihen vor, die zweite die Fachtiefen. Damit lassen sich in den Boxen von Metallform Wächter schnell und einfach Fachungen für die sichere Aufnahme unterschiedlicher Teile bilden.

\section{Mit auswechselbaren Einsätzen Kosten senken}

Diese flexible Lösung ermöglicht es auch, bisher für Schüttgut verwendete Boxen in Warenträger umzugestalten. Alternativ können die Einsätze in Kombination mit einem Grundgestell oder Reinigungskorb in Sonderabmessungen genutzt werden. Die Fixierung der Teileaufnahmen in der Box oder dem Grundgestell erfolgt über gefederte Rastbolzen. Je nach Teilegröße lassen sich in Kombination mit Einlegeböden mehrere Lagen Werkstücke in einer Box platzieren. Um empfindliche Werkstücke vor Beschädigung zu schützen, können die Einsätze beschichtet oder punktuell mit Teflonrohr ummantelt werden. Bei mehrlagiger Bestückung sorgen Kunststoffmatten, die als Zwischenlage eingesetzt werden, für einen Bauteilschutz.

Diese Lösung ermöglicht, dass bei einem Teilewechsel statt des kompletten Reinigungsbehältnisses nur noch die Einsätze zu tauschen sind. Die Box kann weiterverwendet werden, was zu deutlichen Kosteneinsparungen führt.

Mit dem Boxen-System hat Metallform ein aufeinander abgestimmtes Programm von Reinigungskörben entwickelt. Durch diverse Zubehörteile kann es bedarfsgerecht erweitert werden. Das umfangreiche Sortiment an Standardkomponenten, wie Einlege- böden, Verschluss-, Spann- und Auflagendeckel, Fachstangen und -teiler sowie Schüttgutkörbe, wird durch passende Sonderhorden ergänzt. Die Reinigungsbehältnisse können als Tauch-, Hub-, Dreh-, oder Wippkorb eingesetzt werden. Dank ihres stabilen Aufbaus sind Auflasten bis 600 kg möglich. Die Boxen bieten volle Kompatibilität zu handelsüblichen Waren- und Transportkästen.

\section{Lange Lebensdauer und hohe} Sicherheit durch Edelstahl Gefertigt werden die Einsätze sowie alle Komponenten des Box-Systems serienmäßig aus rostfreien Edelstahl-Rundstäben mit elektropolierter Oberfläche. Der runde, glatte Draht gewährleistet die gute Zugänglichkeit der Medien und Waschmechanik, beispielsweise Ultraschall und Injektionsflutwaschen, zu den Teilen. Gleichzeitig werden Verschleppungen minimiert und die Trocknungszeit reduziert. Darüber hinaus verhindert das hochwertige Material Rückverschmutzungen der Teile durch das Behältnis und sorgt für eine lange Lebensdauer der Werkstückträger. Dazu trägt auch die sorgfältige Verarbeitung bei. So erfolgt beispielsweise die Verschweißung stumpf, so dass keine scharfen Ecken und Kanten vorhanden sind.

\section{Kontakt:}

Metallform Wächter GmbH,Bretten,

Tel. 07252942636 ,

joerg.schleeh@metallform.de,

www.metallform.de 